Pacific Journal of Mathematics

NEIGHBORLY BUSHES AND THE RADON-NIKODÝM

PHILIP W. 


\title{
NEIGHBORLY BUSHES AND THE RADON-NIKODÝM PROPERTY FOR BANACH SPACES
}

\author{
Philip W. McCartNey
}

We introduce the neighborly bush property (NBP). A dual Banach space has the NBP if and only if it contains a bush, but there is a Banach space with the NBP that does not contain a bush and therefore has the RadonNikodým property. Any Banach space with the NBP has a nonseparable second dual. Other related results are obtained.

A Banach space $X$ has the Radon-Nikodým property (RNP) if and only if, for every finite measure space $(\Omega, \Sigma, \mu)$, and each $\mu$ continuous, $X$-valued measure $m$ on $\Sigma$ of bounded variation, there exists a Bochner-integrable function $f: \Omega \rightarrow X$ such that

$$
m(E)=\int_{E} f d \mu \text { if } E \text { is in } \Sigma .
$$

For convenience in defining "tree," we introduce the set $S=$ $\left\{(k, j): k \in N, j \in N, 1 \leqq j \leqq 2^{k-1}\right\}$, where $N$ denotes the set of positive integers. Also, for each positive integer $n$, let $S_{n}=\{(k, j) \in S$ : $k \leqq n\}$.

Definition 1. Let $X$ be a Banach space and $\varepsilon$ a positive number. A tree with separation constant $\varepsilon$ is a function $T: S \rightarrow X$ such that, for every $(k, j)$ in $S$,

(i ) $T(k, j)=1 / 2[T(k+1,2 j)+T(k+1,2 j-1)]$

(ii) $\|T(k, j)\| \leqq 1$,

(Averaging Property),

(iii) $\|T(k+1,2 j)-T(k+1,2 j-1)\|>\varepsilon$

(Separation Property).

We denote $T(k, j)$ by $x^{k, j}$, and call $x^{k+1,2 j-1}$ and $x^{k+1,2 j}$ the branch points of $T$ corresponding to the node $x^{k, j}$. The connection between the RNP and trees seems to have been noted first (implicitly) in [9] and explicitly in [11, page 222]. It follows easily from dentability that if a Banach space contains a tree, it does not have the RNP [3, page 127]. A dual space $X$ has the RNP if and only if $X$ does not contain a tree ([3, page 127], [11, page 222]). A Banach space has the RNP if and only if it does not contain a bush [3, page 216]. The reader who is interested in further historical information should see [1], [2], [3], and [5]. There are many other characterizations of the RNP discussed in [1], [2], and [3]. 
In this paper, we will consider a variation of the definition of "tree" (and "bush"). Originally, this was begun in the hope of shedding light on open questions related to the question of whether every Banach space without the RNP contains a tree i.e., whether every Banach space which contains a bush contains a tree. However, the concepts which resulted from these variations have proved to be very interesting in their own right.

A natural and severe weakening of a tree is a "finite tree," which we define next.

Definition 2. Let $X$ be a Banach space, $\varepsilon$ a positive number, and $n$ a positive integer. A finite tree with separation constant $\varepsilon$ is a function $T: S_{n} \rightarrow X$ which satisfies conditions (i)-(iii) of Definition 1 . In that case, we say that $T$ is a finite tree with $n$ columns. We say $X$ has the finite-tree property if and only if there exists a positive number $\varepsilon$ such that for exery positive integer $n, X$ contains a finite tree with $n$ columns and separation constant $\varepsilon$. [7, pages 159-160].

A Banach space $X$ is said to finitely representable in a Banach space $Y$ if, for every finite-dimensional subspace $X_{n}$ of $X$ and every $\lambda>1$, there is an isomorphism $T$ of $X_{n}$ into $Y$ for which $\lambda^{-1}\|x\| \leqq$ $\|T(x)\| \leqq \lambda\|x\|$ if $x \in X_{n}$. A Banach space $B$ is super-reflexive if no nonreflexive Banach space is finitely representable in $B$. In fact, a Banach space is super-reflexive if and only if it does not have the finite-tree property ([7] and [8]), or if and only if it is isomorphic to a uniformly convex space [4].

One should notice that, given a tree $T$ and a positive integer $n$, we can recover the elements $T(k, j)$ for which $1 \leqq k<n$ by averaging back along $S_{n}$ from the appropriate subset of the elements $T(n, j)$ for which $1 \leqq j \leqq 2^{n-1}$. Suppose we are given only the set $\left\{x^{n, j}: 1 \leqq j \leqq 2^{n-1}\right\}$ of elements in the $n$th column of a tree. Then each $x^{k, i}$ with $1 \leqq k<n$ is the average of the elements in the $n$th column which follow $x^{k, i}$ in the tree structure. We can this process backward averaging.

Let $\left\{n_{k}\right\}$ be an increasing sequence of positive integers such that $n_{1}=1$, and $2 \cdot n_{k-1} \leqq n_{k}$ for every positive integer $k$. The set $A=$ $\left\{(k, j): k, j \in N\right.$ and $\left.1 \leqq j \leqq n_{k}\right\}$ is a bush domain. For each positive integer $k$, let $C_{k}=\left\{i \in N: 1 \leqq i \leqq n_{k}\right\}$, let $0_{k}=\{(n, j) \in A: n=k\}$, and let $\boldsymbol{A}_{k}=\{(n, j) \in \boldsymbol{A}: n \leqq k\}$.

Definition 3. Suppose $X$ is a Banach space, $A$ is a bush domain, and $\varepsilon$ is a positive number. Let $S_{1,1}=\{1\}$, and, for every $k \geqq 2$, let $\left\{S_{k, j}: 1 \leqq j \leqq n_{k-1}\right\}$ be a partition of $C_{k}$ into pairwise disjoint sets of consecutive integers. A bush with separation con- 
stant $\varepsilon$ is an ordered pair $(B, P)$ for which $B$ is a function mapping $A$ into the closed unit ball of $X, P=\left\{S_{k, j}:(k, j) \in A\right\}$, and, for every $k \geqq 2$ and every $j$ with $i \leqq j \leqq n_{k-1}$,

(i) $\left|S_{k, j}\right| \geqq 2$,

(ii) $B(k-1, j)=\left(1 /\left|S_{k, j}\right|\right) \sum_{i \in S_{k, j}} B(k, i)$ (Averaging Property),

(iii) for every $(k, j)$ in $A$ and $i$ in $S_{k+1, j}$,

$$
\|B(k, j)-B(k+1, i)\|>\varepsilon \quad \text { (Separation Property). }
$$

We denote $B(k, j)$ by $x^{k, j}$ and call $\left\{x^{k+1, i}: i \in S_{k+1, j}\right\}$ the set of branch points of $(B, P)$ corresponding to the node $x^{k, j}$. In this case we say that $X$ contains the bush $(B, P)$.

If $n$ is a positive integer and $B$ is defined only on $A_{n}$, we suppress the set $P$, and say that $B$ is a finite bush if (i)-(iii) hold on $\boldsymbol{A}_{n}$.

Definition 4. A Banach space $X$ has the neighborly tree property (NTP) if and only if there are positive numbers $\varepsilon$ and $\delta$ for which $\delta<\varepsilon / 4$ and the closed unit ball of $X$ contains a sequence $\left(T_{n}\right)$ of finite trees satisfying:

(i) Each $T_{n}$ is defined on $S_{n}$.

(ii) Each $T_{n}$ has separation constant $\varepsilon$.

(iii) For every $(k, j)$ in $S$, there is a ball in $X$ of radius $\delta$ which contains $\left\{x_{n}^{k, j}: n \geqq k\right\}$, where $T_{n}(k, j)=x_{n}^{k, j}$ when $(k, j) \in S_{n}$. The condition " $\delta<\varepsilon / 4$ " is needed to insure the separation of elements when we construct trees in dual spaces which have the NTP. Clearly, some condition of this type is needed if we want the NTP to be stronger than the finite-tree property.

The neighborly tree property is stronger than the finite-tree property, since there is a reflexive space with the finite-tree property [7], but we will see that a reflexive space cannot have the neighborly tree property. As we shall see in Example 9, there is a Banach space which has the neighborly tree property but which contains no tree.

Definition 5. A Banach space $X$ has the neighborly bush property (NBP) if and only if there is a bush domain $A$, a set $P$ as in Definition 3, and positive numbers $\varepsilon$ and $\delta<\varepsilon / 4$, such that the closed unit ball of $X$ contains a sequence $\left\{B_{n}\right\}$ of finite bushes satisfying:

(i) Each $B_{n}$ is defined on $\boldsymbol{A}_{n}$, the partition of which is determined by $P$.

(ii) Each $B_{n}$ has separation constant $\varepsilon$.

(iii) For every $(k, j)$ in $\boldsymbol{A}$, there is a ball in $X$ of radius $\delta$ which contains $\left\{x_{n}^{k, j}: n \geqq k\right\}$, where $B_{n}(k, j)=x_{n}^{k, j}$ when $(k, j) \in \boldsymbol{A}_{n}$. 
In Theorems 10 and 11, we will see how a space with the NBP is related to a space containing a bush.

A Banach space with the NTP has the NBP, but it is not known whether a Banach space with the NBP also has the NTP. That problem seems to be related to the unsolved problem of whether a Banach space which contains a bush also contains a tree.

The fact that the closed unit ball of a reflexive space is weaksequentially compact, together with a diagonalization argument, can be used to show that if a reflexive space has the NBP, then it contains a bush. But every reflexive space has the RNP ([3, page 218] and [10, page 134]), and therefore contains no bush [3, page 216]. Hence, no reflexive space has the NBP. Likewise, $\omega^{*}$-sequential compactness of the closed unit ball of the dual of a separable space can be used to show that if $X$ is a separable Banach space whose dual $X^{*}$ has the NBP (NTP), then $X^{*}$ contains a bush (tree). Since every separable dual has the RNP [2, page 5], we conclude that no separable dual has the NBP (NTP). In fact, as we will see in Theorem 6, any dual space $X^{*}$ with the NBP (NTP) contains a bush (tree) whether or not $X$ is separable.

Although the suggested method of proving that no reflexive space has the NBP (NTP) is rather intuitive, this result is a corollary of Theorem 6. For suppose $X$ is reflexive and has NBP. Then $Y$, the closed linear span of the elements of a particular neighborly bush structure, is reflexive. But $Y^{* *}$ has the NBP and therefore, by Theorem 6, $Y^{* *}$ contains a bush. Since $Y$ is reflexive and no reflexive space contains a bush, the presence of a bush in $Y^{* *}$ is a contradiction.

Since the natural image of $X$ in $X^{* *}$ inherits the NBP from $X$, and since no separable dual has the NBP, it follows that $X^{* *}$ is not separable if $X$ has the NBP.

If a Banach space $X$ does not have the NTP, then $X$ does not contain a tree. Hence, if $X$ is also a dual, then $X$ has the RNP [11, page 222]. Likewise, if a Banach space does not have the NBP, then it does not contain a bush, and therefore has the RNP [3, page 216].

Theorem 6. If $X$ is a Banach space whose dual $X^{*}$ has the NBP (NTP), then $X^{*}$ contains a bush (tree).

Since the proof of Theorem 6 very closely parallels the proof that (ii) implies (iii) in Theorem 7, we will only outline the proof of Theorem 6. Let $\left(B_{n}\right)$ be a sequence of finite bushes in $X^{*}$, satisfying (i)-(iii) of Definition 5, with $\varepsilon>0,0<\delta<\varepsilon / 4$, and bush domain $A$. For each $(k, j)$ in $A_{n}$, denote $B_{n}(k, j)$ by $f_{n}^{k, j}$. For every 
$(k, j)$ in $A$, let $B^{k, j}$ be a closed ball of radius $\delta$ with the property that, for every $n$ in $N, f_{n}^{k, j}$ is in $B^{k, j}$. Denote the center of $B^{k, j}$ by $g^{k, j}$. We give each $B^{k, j}$ the relative $\omega^{*}$-topology, so that the topological product $Q=\Pi_{A} B^{k, j}$ is compact.

For every $n$ in $N$, define an element $\omega_{n}$ in $Q$ by:

$$
\omega_{n}(k, j)= \begin{cases}f_{n}^{k, j} & \text { if } k \leqq n, \\ g^{k, j} & \text { if } k>n .\end{cases}
$$

Since $Q$ is countably compact, the sequence $\left(\omega_{n}\right)$ has a cluster point $\omega$ in $Q$. It can be shown that $(\omega, P)$ is a bush in $X^{*}$.

THeOREM 7. If $X$ is a Banach space, then the following statements are equivalent.

(i) $X^{*}$ has the NBP (NTP).

(ii) $X$ has a separable subspace $Y$ such that $Y^{*}$ contains a bush (tree).

(iii) $X^{*}$ contains a bush (tree).

Proof. Let us prove first that (i) implies (ii). Let $\left(B_{n}\right)$ be a sequence of finite bushes and $A$ a bush domain exhibiting the NBP in $X^{*}$. Let $W$ be the collection of all bush elements occurring in any of the finite bushes $B_{n}$. For every $\theta$ such that $0<\theta<1$, we define a subset $C_{\theta}$ of the closed unit ball $U$ of $X$, by letting $C_{\theta}=\left\{x_{n}^{k j}\right.$ : $n \in N$ and $\left.(k, j) \in A_{n}\right\}$, where each $x_{n}^{k, j}$ is chosen so that $f_{n}^{k, j}\left(x_{n}^{k, j}\right) \geqq \theta$. $\left\|f_{n}^{k, j}\right\|$. For every $i$ in $S_{k, j}$ and $n$ in $N$, choose $y_{n}^{k, i}$ in $U$ with the property that

$$
\left|\left(f_{n}^{k, i}-f_{n}^{k-1, j}\right)\left(y_{n}^{k, i}\right)\right| \geqq \theta \cdot\left\|f_{n}^{k, i}-f_{n}^{k-1, j}\right\| .
$$

Let $B_{\theta}=\left\{y_{n}^{k, i}: n \in N\right.$ and $\left.(k, i) \in A_{n}\right\}$ and $D=\bigcup_{i=1}\left(C_{(i-1) / i} \cup B_{(i-1) / i}\right)$. Then $D \subseteq U$ is countable and:

(i) $D$ is norming for $W$,

(ii) If $Y$ is the closed linear span of $D$, then $Y$ is a separable subspace of $X$ such that, if $g_{n}^{k, j}$ is the restriction of $f_{n}^{k, j}$ to $Y$, then $\left\|g_{n}^{k, j}\right\|=\left\|f_{n}^{k, j}\right\|$ and, for every $i$ in $S_{k, j},\left\|g_{n}^{k-1, j}-g_{n}^{k, i}\right\|=\left\|f_{n}^{k-1, j}-f_{n}^{k, i}\right\|$. Thus, $\left\{\left(g_{n}^{k, j}\right)_{A_{n}}: n \in N\right\}$ forms a neighborly bush structure in $Y^{*}$. This and the fact that $Y$ is separable imply $Y^{*}$ contains a bush.

We now prove that (ii) implies (iii). Let $(B, P)$ be a bush in $Y^{*}$ with bush domain $A$, and separation constant $\varepsilon>0$. Denote $B(k, j)$ by $g^{k, j}$ for each $(k, j)$ in $A$, and use the Hahn-Banach theorem to extend $g^{k, j}$ to an element $f^{k, j}$ in $X^{*}$ with the same norm as $g^{k, j}$. Next, let $U^{0}$ be the closed unit ball of $X^{*}$, with the relative $\omega^{*}$ topology. Then the topological product $Q=\Pi_{A} U^{0}$ is compact.

For every $n$ in $N$, define an element $\omega_{n}$ in $Q$ as follows. Let 
$\omega_{n}(k, j)=\bar{O}$ if $n<k, \omega_{n}(n, j)=f^{n, j}$, and if $n>k$ define $\omega_{n}(k, j)$ by averaging back from the $n$th column. For example, for every $(2, j)$ in $A, \omega_{3}(2, j)=\left(1 /\left|S_{3, j}\right|\right) \sum_{i \in S_{3, j}} f^{3, i}$, and

$$
\omega_{3}(1,1)=\frac{1}{\left|S_{2,1}\right|} \sum_{i \in S_{2,1}} \omega_{3}(2, i) .
$$

Since $Q$ is countably compact, the sequence $\left(\omega_{n}\right)$ has a cluster point $\omega \in Q$. We will show that $(\omega, P)$ is a bush in $X^{*}$.

Since for every $(k, j)$ in $A$, the projection map $\Pi_{k, j}$ of $Q$ onto $U^{0}$ is continuous, we have that $\omega(k, j)$ is a $\omega^{*}$-cluster point of $\left(\omega_{n}(k, j)\right)_{n}$. But $U^{0}$ is $\omega^{*}$-closed and therefore $\omega(k, j)$ is in $U^{0}$.

Fix $(k, j)$ in $A$. The definition of $\omega_{n}$ implies that for every $s \geqq k$ and for every $y$ in $Y, \omega_{s}(k, j)(y)=g^{k, j}(y)$. But if $\eta$ is positive and $y$ is $Y$, then there is an $s \geqq k$ such that $\left|\omega_{s}(k, j)(y)-\omega(k, j)(y)\right|<\eta$. Hence, $\left|g^{k, j}(y)-\omega(k, j)(y)\right|<\eta$. Since $\eta$ was an arbitrary positive number, we have $g^{k, j}(y)=\omega(k, j)(y)$, and therefore $g^{k, j}$ is the restriction of $\omega(k, j)$ to $Y$. Thus, for every $i$ in $S_{k+1, j},\|\omega(k, j)-\omega(k+1, i)\| \geqq$ $\left\|g^{k, j}-g^{k+1, i}\right\|>\varepsilon$.

To see that the averaging property holds, fix $x$ in $X$, let $\sigma$ be a positive number and choose $(k-1, j)$ in $A$. For every $(r, s)$ in $A$, let $V(r, s)=\left\{g \in U^{0}:|g(x)-\omega(r, s)(x)|<\sigma\right\}$. We denote that the set

$$
\theta=\Pi_{k-1, j}^{\overleftarrow{ }}(V(k-1, j)) \cap \bigcap_{i \in S_{k, j}} \Pi_{k, i}^{\overleftarrow{ }}(V(k, i))
$$

is a $Q$-neighborhood of $\omega$. Therefore, since $\omega$ is a $Q$-cluster point of $\left(\omega_{n}\right)$, there is an $s \geqq k$ such that $\omega_{s}$ is in $\theta$. By the definition of $\omega_{s}$, we have

$$
\omega_{s}(k-1, j)(x)=\frac{1}{\left|S_{k, j}\right|} \sum_{i \in S_{k, j}} \omega_{s}(k, i) .
$$

Since $\omega_{s}$ is in $\bigcap_{i \in S_{k, j}} \prod_{k, i}^{\leftarrow}(V(k, i))$, we have, for every $i$ in $S_{k, j}$,

$$
\left|\omega_{s}(k, i)(x)-\omega(k, i)(x)\right|<\sigma .
$$

Thus, $\left|\sum \omega_{s}(k, i)(x)-\sum \omega(k, i)(x)\right|<\left|S_{k, j}\right| \cdot \sigma$, where both sums are over $S_{k, j}$. By equation (1),

$$
|| S_{k, j}\left|\cdot \omega_{s}(k-1, j)(x)-\sum \omega(k, i)(x)\right|<\left|S_{k, j}\right| \cdot \sigma .
$$

But $\omega_{s}$ is in $\Pi_{k-1, j}(V(k-1, j))$, and therefore

$$
|| S_{k, j}\left|\cdot \omega_{s}(k-1, j)(x)-\right| S_{k, j}|\cdot \omega(k-1, j)(x)|<\left|S_{k, j}\right| \cdot \sigma .
$$

Hence, by (2) and (3),

$$
|| S_{k, j}\left|\cdot \omega(k-1, j)(x)-\sum \omega(k, i)(x)\right|<2 \sigma \cdot\left|S_{k, j}\right|,
$$


where the sum is over $S_{k, j}$. But $\sigma>0$ and $x$ in $X$ were arbitrary. Therefore,

$$
\omega(k-1, j)=\frac{1}{\left|S_{k, j}\right|} \sum_{i \in S_{k i}} \omega(k, i) .
$$

Since (iii) formally implies (i), the proof of Theorem 7 is complete.

The next result also appears as a special case of the main theorem in [11].

CoRollary 8. If a Banach space $X$ has a subspace $Y$ isomorphic to $l_{1}$, then $X^{*}$ contains a tree.

It is not true in general that if $X$ has the NTP, then $X^{*}$ has the NBP. For example, $c_{0}$ contains a tree and $c_{0}^{*}=l_{1}$, which does not have the NBP. Furthermore, it is not true in general that if $X^{*}$ has the NTP, then $X$ has the NBP. For example, $l_{\infty}$ contains a tree, and is the dual of $l_{1}$.

It is also not true in general that if $X^{* *}$ has the NTP, then $X$ has the NBP. In fact, $l_{1}$ does not have the NBP, but by Corollary $8 l_{1}^{* *}=l_{\infty}^{*}$, the space of all finitely additive measures on the algebra of all subsets of $N$, contains a tree.

In view of the discussion preceding Theorem 6, we might ask whether a Banach space $X$, whose dual $X^{*}$ is separable and whose second dual $X^{* *}$ is nonseparable, must have the NBP? The answer is no. In fact, if $B$ is a Banach space such that $B^{* *}$ is separable and $B^{* * *}$ is nonseparable, then $X=B^{*}$ is a separable dual, and therefore does not have the NBP. The existence of such a space $B$ was established in 1960 by R. C. James [6].

EXAMPLE 9. We construct a Banach space that has the NTP, but which contains no bush. By the remarks preceding Theorem 6 , no such space can be a dual. Our example also shows that a Banach space with the NTP need not contain a tree, and can have the RNP.

For every $j \in N$, let $e_{j}$ be the member of $c_{0}$ for which

$$
e_{j}(n)=\left\{\begin{array}{lll}
1 & \text { if } & n=j \\
0 & \text { if } & n \neq j
\end{array}\right.
$$

Let $T=\left(t^{k, j}\right)_{S}$ be the tree in $c_{0}$ whose elements are: $t^{1,1}=e_{1}, t^{2,1}=$ $e_{1}+e_{2}, t^{2,2}=e_{1}-e_{2}, t^{3,1}=e_{1}+e_{2}+e_{3}, t^{3,2}=e_{1}+e_{2}-e_{3}, t^{3,3}=e_{1}-e_{2}+e_{3}$ $t^{3,4}=e_{1}-e_{2}-e_{3}$, etc. Let $P=c_{0} \times{ }_{l_{1}} \Pi_{l_{1}} l_{\infty}^{(n)}$, where the $l_{1}$-norm is used in defining the product norms, and $l_{\infty}^{(n)}$ denotes the space of real-valued functions on $\{1,2, \cdots, n\}$ with the sup norm. Let $\left(t_{n}^{k, j}\right)_{S_{n}}$ 
denote the finite tree in $l_{\infty}^{(n)}$ obtained from $T$ by truncating each $t^{k, j}$ to get an element of $l_{\infty}^{(n)}$.

Fix $\alpha$ with $3 / 4<\alpha<1$. For every $n$ in $N$, let $T_{n}=\left(x_{n}^{k, j}\right)_{S_{n}}$ be the finite tree of $n$ columns in $P$ whose elements are defined as follows. For every $(k, j) \in S_{n}, x_{n}^{k, j} \in P$ has only two nonzero components; its $c_{0}$ component is $\alpha \cdot t^{k, j}$, and its $l_{\infty}^{(n)}$ component is $(1-\alpha) t_{n}^{k, j}$. Thus, $\left\|x_{n}^{k, j}\right\|=\alpha+(1-\alpha)=1$. If $j$ is odd, then

$$
\begin{aligned}
\left\|x_{n}^{k, j}-x_{n}^{k, j+1}\right\| & =\alpha \cdot\left\|t^{k, j}-t^{k, j+1}\right\|+(1-\alpha)\left\|t_{n}^{k, j}-t_{n}^{k, j+1}\right\| \\
& =2 \alpha+(1-\alpha) \cdot 2=2 .
\end{aligned}
$$

Further, if $j$ is odd and $k \geqq 2$, then

$$
\frac{1}{2}\left(x_{n}^{k, j}+x_{n}^{k, j+1}\right)=x_{n}^{k-1,(j+1) / 2} .
$$

To see that the sequence $\left\{T_{n}\right\}$ exhibits the NTP in $P$, we need only notice that $\varepsilon=2$ and, if $(k, j)$ is in $S$ and $m>n \geqq k$, then

$$
\left\|x_{m}^{k, j}-x_{n}^{k, j}\right\|=0+(1-\alpha)+(1-\alpha)=2-2 \alpha<\frac{1}{2}=\frac{\varepsilon}{4} .
$$

Let $V$ be the set of all elements of $P$ that appear in any of the $T_{n}$ 's. Let $Y$ be the closed linear span of $V$. Then $Y$ has the NTP, but contains no tree.

That $Y$ has the NTP has been shown. To see that $Y$ does not contain a tree, it suffices to show that if $Y$ did contain a tree, then the separable dual $\Pi_{l_{1}} l_{\infty}^{(n)}=\left(\prod_{c_{0}} l_{\infty}^{(n)}\right)^{*}$ would contain a tree.

Let $z \in \operatorname{sp}(V)$ and represent $z$ as $\sum_{i=1}^{M} c_{i} \cdot x_{n(i)}^{k(i), j(i)}$. For every $x_{n}^{k, j}$ in $V$, let $\hat{x}_{n}^{k, j}$ be the element of $P$ whose $l_{\infty}^{(n)}$ component is $(1-\alpha) t_{n}^{k, j}$, and all of whose other components are zero vectors. We will express explicitly only the $c_{0}$ and any nonzero $l_{\infty}^{(n)}$ components of the elements of $P$, and write $x_{n(i)}^{k(i), j(i)}$ as $\left[\alpha t^{k, j},(1-\alpha) t_{n}^{k, j}\right]_{i}$, and $\hat{x}_{n(i)}^{k(i), j(i)}$ as $\left[\bar{O},(1-\alpha) t_{n}^{k, j}\right]_{i}$, where the subscript $i$ denotes that $t_{n}^{k, j}$ should be $t_{n(i)}^{k(i), j(i)}$. Let $K=\max \left\{n_{i}: 1 \leqq i \leqq M\right\}$ and map each $t_{n}^{k, j}$ naturally into $l_{\infty}^{(K)}$, denoting its image by $\widetilde{t}_{n}^{k, j}$. Note that since the $l_{1}$ norm was used as the product norm for $P$,

$$
\begin{aligned}
\|z\| & =\left\|\sum_{i=1}^{M} c_{i} \cdot\left[\alpha t^{k, j},(1-\alpha) t_{n}^{k, j}\right]_{i}\right\| \\
& =\left\|\sum_{i=1}^{M} c_{i} \cdot\left[\alpha t^{k, j}, \bar{O}\right]_{i}\right\|+\left\|\sum_{i=1}^{M} c_{i} \cdot\left[\bar{O},(1-\alpha) t_{n}^{k, j}\right]_{i}\right\| \\
& =\alpha \cdot\left\|\sum_{i=1}^{M} c_{i}\left[t^{k, j}, \bar{O}\right]_{i}\right\|+\left\|\sum_{i=1}^{M} c_{i} \cdot\left[\bar{O},(1-\alpha) t_{n}^{k}{ }^{j}\right]_{i}\right\| .
\end{aligned}
$$

But 


$$
\begin{aligned}
& \left\|\sum_{i=1}^{M} c_{i}\left[\bar{O},(1-\alpha) t_{n}^{k, j}\right]_{i}\right\| \geqq\left\|\sum_{i=1}^{M} c_{i}\left[\bar{O},(1-\alpha) \widetilde{t}_{n}^{k, j}\right]_{i}\right\| \\
& =(1-\alpha) \cdot\left\|\sum_{i=1}^{M} c_{i}\left[\bar{O}, \tilde{t}_{n}^{k, j}\right]_{i}\right\|=(1-\alpha) \cdot\left\|\sum_{i=1}^{M} c_{i}\left[t^{k, j}, \bar{O}\right]_{i}\right\| .
\end{aligned}
$$

Hence, $\|z\| \leqq((\alpha / 1-\alpha)+1) \cdot\left\|\sum_{i=1}^{M} c_{i}\left[\bar{O},(1-\alpha) t_{n}^{k, j}\right]_{i}\right\|$, and therefore

$$
(1-\alpha)\left\|\sum_{i=1}^{M} c_{i} \cdot x_{n(i)}^{k(i), j(i)}\right\| \leqq\left\|\sum_{i=1}^{M} c_{i} \cdot \hat{x}_{n(i)}^{k(i), j(i)}\right\| .
$$

If $Y$ did contain a tree, then by making the $c_{0}$ component of each tree element $\bar{O}$, we would obtain a tree in $\Pi_{l_{1}} l_{\infty}^{(n)}$; for the averaging property would clearly hold, the induced tree is composed of elements of norm $\leqq 1$, and inequality (1) implies that the separation property is transferred to the induced tree.

Since $\Pi_{l_{1}} l_{\infty}^{(n)}$ has the RNP, it contains no bush. Thus, $Y$ contains no bush; for if $Y$ did contain a bush, then inequality (1) together with the observations that the averaging property is maintained, and that the new elements stay within the unit ball, would yield a bush in $\Pi_{l_{1}} l_{\infty}^{(n)}$.

Since $Y$ contains no bush, it has the RNP. Thus we have shown that a Banach space can have the NBP and not contain a bush.

The next two theorems describe a sense of nearness for which every Banach space having the NBP is near a bush in some Banach space.

TheOREM 10. Suppose $M$ and $W$ are subspaces of a Banach space $X$, and that $M$ contains a bush (tree) $(B, P)$ with separation constant $\sigma$. If $0<\delta<\sigma / 6$ and every element of $B$ is within $\delta$ of $W$, then $W$ has the NBP (NTP).

Proof. For every $(k, j)$ in $A$, let $x^{k, j}=B(k, j)$, and for every $n$ in $\boldsymbol{N}$ and $(n, j)$ in $A$, choose $y_{n}^{n, j}$ in $W$ such that $\left\|y_{n}^{n, j}-x^{n, j}\right\|<\delta$ and $\left\|y_{n}^{n, j}\right\| \leqq 1$. For every $(k, j)$ in $A_{n}$, define $y_{n}^{k, j}$ by backward averaging from the $y_{n}^{n, j}$ 's, that is, for every $(k, j)$ in $A_{n}$,

$$
y_{n}^{k-1, j}=\sum_{i \in S_{k}, j} \frac{1}{\left|S_{k, j}\right|} y_{n}^{k, j} \text {. }
$$

It then follows that, for every $(k, j)$ in $A_{n},\left\|x^{k, j}-y_{n}^{k, j}\right\|<\delta$.

To see that the separation property holds, we notice that, if $(k-1, j)$ is in $A$ and $i$ is in $S_{k, j}$, then

$$
\begin{aligned}
\sigma & <\left\|x^{k, i}-x^{k-1, j}\right\| \\
& \leqq\left\|x^{k, i}-y_{n}^{k, i}\right\|+\left\|y_{n}^{k, i}-y_{n}^{k-1, j}\right\|+\left\|y_{n}^{k-1, j}-x^{k-1, j}\right\| \\
& \leqq 2 \delta+\left\|y_{n}^{k, i}-y_{n}^{k-1, j}\right\|,
\end{aligned}
$$


for every $n \geqq k$. Hence, $\sigma-2 \delta<\left\|y_{n}^{k, i}-y_{n}^{k-1, i}\right\|$ whenever $n \geqq k$. Let $\varepsilon=\sigma-2 \delta$. Then $0<\delta<\varepsilon / 4$, since $\delta<\sigma / 6$. Thus $W$ has the NBP.

For the next theorem, we assume that the Banach space $X$ has a norming sequence of linear functionals. This assumption is valid when $X$ is separable, or if $X$ is any subspace of $l_{\infty}$.

THeOREM 11. Suppose $X$ is a Banach space with the NBP (NTP), and $\varepsilon$ and $\delta$ are as in Definition 5. If $X$ has a norming sequence of linear functionals, then $X$ can be embedded in a Banach space containing a bush (tree), in such a way that every bush (tree) element is within $\delta$ of $X$.

Proof. Let $\left\{\left(x_{n}^{k, j}\right)_{A_{n}}: n \in N\right\}$ exhibit the NBP in $X$, and for every $(k, j)$ in $A$, let $x^{k, j}$ in $X$ be such that, for every $n \geqq k,\left\|x^{k, j}-x_{n}^{k, j}\right\|<\delta$. Let $P=\Pi_{N} X$ and define $y^{1,1}$ in $P$ by letting $y_{(n)}^{1,1}=x_{n}^{1,1}$ for every $n$ in $N$. For every $(2, j)$ in $A$, let $y_{(1)}^{2, j}=x_{1}^{1,1}$ and $y_{(n)}^{2, j}=x_{n}^{2, j}$, if $n \geqq 2$. In general, if $(k, s)$ is in $A$ and $s \in S_{k, j}$, we let

$$
y_{(i)}^{k, s}= \begin{cases}y_{(i)}^{k, 1, j} & \text { if } i<k, \\ x_{i}^{k, s} & \text { if } i \geqq k\end{cases}
$$

It follows easily that

$$
y^{k-1, j}=\sum_{i \in S_{k}, j} \frac{1}{\left|S_{k, j}\right|} \cdot y^{k, i}
$$

Let $\left(f_{n}\right)$ be a norming sequence of linear functionals for $X$, each of which has norm one. For every positive integer $n$ and every $(k, j)$ in $A$, the sequence $\left\{f_{n}\left(y_{\langle i\rangle}^{k, j}\right)\right\}_{i}$ is bounded, and therefore since $N \times A$ is countable, we can diagonalize to get a strictly increasing sequence of positive integers $\left\{p_{i}\right\}$, such that for every $n$ in $N$ and every $(k, j)$ in $A, \lim _{i \rightarrow \infty} f_{n}\left(y_{\left(p_{i}\right)}^{k, j}\right)$ exists.

We can identify $X$ with the collection $W$ of constant elements of $P$. Let $Y=\operatorname{span}\left[W \cup\left\{y^{k, j}:(k, j) \in A\right\}\right]$. For every $n$ in $N$ and every $y$ in $Y, \lim _{i \rightarrow \infty} f_{n}\left(y\left(p_{i}\right)\right)$ exists. Therefore it makes sense to define

$$
\text { || } y|\||=\sup _{n} \lim _{i \rightarrow \infty}\left|f_{n}\left(y\left(p_{i}\right)\right)\right|,
$$

for every $y$ in $Y$. In fact, $\||\cdot \||$ is a semi-norm on $Y$.

If $(k, j)$ is in $A$, and $n$ and $i$ are positive integers, then $\left.\left|f_{n}\left(y_{\left(p_{i}\right)}^{k, j}\right)\right| \leqq\left\|f_{n}\right\| \cdot \| y_{\left(p_{i}\right)}^{k, j}\right) \| \leqq 1$. Hence, $\sup _{n}\left\{\lim _{i \rightarrow \infty}\left|f_{n}\left(y_{\left(p_{i}\right)}^{k, j}\right)\right|\right\} \leqq 1$. That is, $\left\|\mid y^{k, j}\right\| \| \leqq 1$.

To see that $\sup _{(k, j) \in A} \operatorname{dist}\left(W, y^{k, j}\right) \leqq \delta$, fix $(k, j)$ in $A$, and let 
$w^{k, j}$ in $W$ have $x^{k, j}$ in all its components. Then it follows from (1) that

$$
\begin{aligned}
|| w^{k, j}-y^{k, j}|| \mid & =\sup _{n} \lim _{i \rightarrow \infty}\left|f_{n}\left(x^{k, j}-y_{\left(p_{i}\right)}^{k, j}\right)\right|, \\
& =\sup _{n} \lim _{i \rightarrow \infty}\left|f_{n}\left(x^{k, j}-x_{p_{i}}^{k, j}\right)\right|<\delta,
\end{aligned}
$$

since for every pair of positive integers $n$ and $i$,

$$
\left|f_{n}\left(x^{k, j}-x_{p_{i}}^{k, j}\right)\right| \leqq\left\|f_{n}\right\| \cdot\left\|x^{k, j}-x_{p_{i}}^{k, j}\right\|<\delta .
$$

The separation property will be established next. Fix $(k-1, j)$ in $A$ and $i$ in $S_{k, j}$. If $n \geqq k$, then

$$
\begin{aligned}
\varepsilon & <\left\|x_{n}^{k, i}-x_{n}^{k-1, j}\right\| \\
& \leqq\left\|x_{n}^{k, i}-x^{k, i}\right\|+\left\|x^{k, i}-x^{k-1, j}\right\|+\left\|x^{k-1, j}-x_{n}^{k-1, j}\right\| \\
& <\left\|x^{k, i}-x^{k-1, j}\right\|+2 \delta,
\end{aligned}
$$

and therefore $\varepsilon-2 \delta<\left\|x^{k, i}-x^{k-1, j}\right\|$. Since $\left\{f_{n}\right\}$ is norming for $X$, we can choose an $f_{m}$ such that $\left|f_{m}\left(x^{k, i}-x^{k-1, j}\right)\right|>\varepsilon-2 \delta$. Then, for every $n \geqq k$, it follows from the definition of the $x^{p, q}$ 's and $\left\|f_{m}\right\|=1$ that

$$
\begin{aligned}
\varepsilon-2 \delta & <\left|f_{m}\left(x^{k, i}-x^{k-1, j}\right)\right| \\
& \leqq\left|f_{m}\left(x^{k, i}-x_{n}^{k, i}\right)\right|+\left|f_{m}\left(x_{n}^{k, i}-x_{n}^{k-1, j}\right)\right|+\left|f_{m}\left(x_{n}^{k-1, j}-x^{k-1, j}\right)\right| \\
& <\delta+\left|f_{m}\left(x_{n}^{k, i}-x_{n}^{k, i}-x_{n}^{k-1, j}\right)\right|+\delta .
\end{aligned}
$$

Hence, $\varepsilon-4 \delta<\left|f_{m}\left(x_{n}^{k, i}-x_{n}^{k-1, j}\right)\right|$ for every $n \geqq k$, and so ||$y^{k, i}-$ $y^{k-1, j} \mid \| \geqq \varepsilon-4 \delta$. Therefore, if $N=\{y \in Y:\|y\|=0\}$, then $Y \cdot \mid N$ is the required space.

\section{REFERENCES}

1. J. Diestel, Geometry of Banach Spaces-Selected Topics, Lecture Notes in Math., 458, Springer-Verlag, Berlin and New York, 1975.

2. J. Diestel and J. J. Uhl, Jr., The Radon-Nikodym theorem for Banach space-valued measures, Rocky Mountain Math. J., 6 (1976), 1-46.

3. - Vector measures, Amer. Math. Soc. Math. Survey's \#15, (1977).

4. P. Enflo, Banach spaces which can be given an equivalent uniformly convex norm, Israel J. Math., 13 (1972), 281-288.

5. R. E. Huff and P. D. Morris, Geometric characterizations of the Radon-Nikodym property in Banach spaces, Studia Math., 56 (1976), 157-164.

6. R. C. James, Separable conjugate spaces, Pacific J. Math., 10 (1960), 563-571.

7. - Some self-dual properties of normed linear spaces, Symposium of InfiniteDimensional, Topology (1967), Annals of Math. Studies, 69 (1972), 159-175.

8. - Super-reflexive Banach spaces, Canad. J. Math., 24 (1972), 896-904.

9. H. B. Maynard, A geometrical characterization of Banach spaces having the RadonNikodym property, Trans. Amer. Math. Soc., 185 (1973), 493-500.

10. R. S. Phillips, On weakly compact subsets of a Banach space, Amer. J. Math., 65 (1943), 108-136. 
11. C. Stegall, The Radon-Nikodym property of conjugate Banach spaces, Trans. Amer. Math. Soc., 206 (1975), 213-223.

Received October 18, 1978 and in revised form June 5, 1979. This paper is part of a Ph. D. dissertation written with the supervision and assistance of R. C. James.

NORTHERN KENTUCKY UNIVERSITY

Highland Heights, KY 41076 


\section{PACIFIC JOURNAL OF MATHEMATICS}

\section{EDITORS}

DONALD BABBITT (Managing Editor)

University of Galifornia

Los Angeles, California 90024

HUGo RossI

University of Utah

Salt Lake City, UT 84112

C. C. MOORE AND ANDREW OGG

University of California

Berkeley, CA 94720

\section{J. DUGUNDJI}

Department of Mathematics University of Southern California Los Angeles, California 90007

R. FINN AND J. MILGRAM Stanford University Stanford, California 94305

ASSOCIATE EDITORS
E. F. BECKENBACH
B. H. NeumanN
F. WoLF
K. YosHIDA

\section{SUPPORTING INSTITUTIONS}

UNIVERSITY OF BRITISH COLUMBIA UNIVERSITY OF SOUTHERN CALIFONIA CALIFORNIA INSTITUTE OF TECHNOLOGY UNIVERSITY OF CALIFORNIA MONTANA STATE UNIVERSITY STANFORD UNIVERSITY UNIVERSITY OF HAWAII UNIVERSITY OF TOKYO UNIVERSITY OF NEVADA, RENO UNIVERSITY OF UTAH NEW MEXICO STATE UNIVERSITY WASHINGTON STATE UNIVERSITY OREGON STATE UNIVERSITY UNIVERSITY OF OREGON UNIVERSITY OF WASHINGTON 


\section{Pacific Journal of Mathematics \\ Vol. 87, No. $1 \quad$ January, 1980}

Spiros Argyros, A decomposition of complete Boolean algebras ..........

Gerald A. Beer, The approximation of upper semicontinuous multifunctions

by step multifunctions . . . . ....................

Ehrhard Behrends and Richard Evans, Multiplicity theory for Boolean

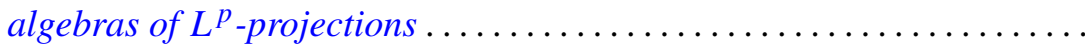

Man-Duen Choi, The full $C^{*}$-algebra of the free group on two

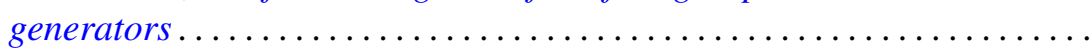

Jen-Chung Chuan, Axioms for closed left ideals in a $C^{*}$-algebra . . . . . . . .

Jo-Ann Deborah Cohen, The strong approximation theorem and locally

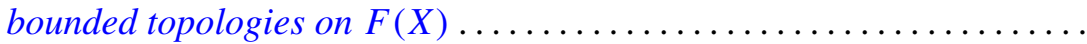

Eugene Harrison Gover and Mark Bernard Ramras, Increasing sequences of Betti numbers............................

Morton Edward Harris, Finite groups having an involution centralizer with

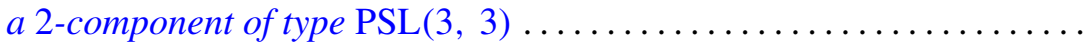

Valéria Botelho de Magalhães Iório, Hopf $C^{*}$-algebras and locally compact

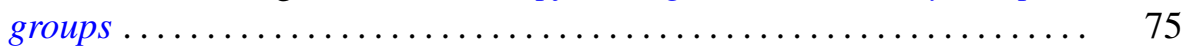

Roy Andrew Johnson, Nearly Borel sets and product measures . . . . . . . . . .

Lowell Edwin Jones, Construction of $Z_{p}$-actions on manifolds . . . . . . . . .

Manuel Lerman and Robert Irving Soare, $d$-simple sets, small sets, and

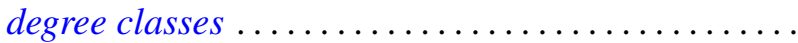

Philip W. McCartney, Neighborly bushes and the Radon-Nikodým property

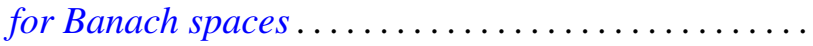

Robert Colman McOwen, Fredholm theory of partial differential equations on complete Riemannian manifolds.

Ernest A. Michael and Carl Preston Pixley, A unified theorem on continuous selections.

Ernest A. Michael, Continuous selections and finite-dimensional sets .

Vassili Nestoridis, Inner functions: noninvariant connected components...

Bun Wong, A maximum principle on Clifford torus and nonexistence of proper holomorphic map from the ball to polydisc.

Steve Wright, Similarity orbits of approximately finite $C^{*}$-algebras . . .

Kenjiro Yanagi, On some fixed point theorems for multivalued

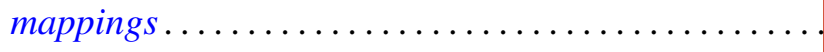

Wieslaw Zelazko, A characterization of LC-nonremovable ideals in commutative Banach algebras 